\title{
Mortality and guideline-concordant care for older patients with schizophrenia: a retrospective longitudinal study
}

Jack Y Tsan ${ }^{1,2^{*}}$, Eileen M Stock ${ }^{3,4}$, Jazmin M Gonzalez ${ }^{5}$, David S Greenawalt ${ }^{1,3}$, John E Zeber ${ }^{3,4}$, Emran Rouf $f^{6}$ and Laurel A Copeland ${ }^{3,4}$

\begin{abstract}
Background: Schizophrenia is associated with excess mortality and multimorbidity, which is possibly associated with difficulty in coordinating care for multiple mental and physical comorbidities. We analyzed the receipt by patients with schizophrenia of 11 types of guideline-concordant care and the association of such care with survival.

Methods: Guideline-concordant care over an 8-year period (financial years 2002 to 2009) was examined in a nationwide sample of 49,173 male veterans with schizophrenia, who were aged 50 years or older. Administrative databases from the electronic medical record system of the Veterans Health Administration (VA) provided comprehensive measures of patient demographics and medical information. Relying on the 2004 American Psychiatric Association guidelines, patterns in 11 types of care were identified and cluster-analyzed. Care types included cardiovascular, metabolic, weight management, nicotine dependence, infectious diseases, vision, and mental health counseling (individual, family, drugs/alcohol, psychiatric medication, and compensated work therapy). Survival analysis estimated association of care patterns with survival, adjusting for clinical and demographic covariates.
\end{abstract}

Results: There was an average of four chronic diseases in addition to schizophrenia in the cohort, notably hypertension (43\%) and dyslipidemia (29\%). Three longitudinal trajectories (clusters) were identified: 'highconsistent' (averaging 5.4 types of care annually), 'moderate-consistent' (averaging 3.8), and 'poor-decreasing' (averaging 1.9). Most veterans were receiving cardiovascular care (67 to 76\%), hepatic and renal function assays (79 to $84 \%$ ), individual counseling (72 to $85 \%$ ) and psychiatry consults (66 to $82 \%$ ), with the proportion receiving care varying by cluster group. After adjustment for age, baseline comorbidity, and other covariates, there was a greater survival rate for those with poor-decreasing care compared with high-consistent care, and for high-consistent compared with moderate-consistent care.

Conclusions: Relatively low levels of guideline-concordant care were seen for older VA patients with schizophrenia, and trajectories of care over time were associated with survival in a non-intuitive pattern. The group with the lowest and decreasing levels of care was also the oldest, but nonetheless had the best age-adjusted and other covariate-adjusted survival rates, possibly because they were requiring less care relative to younger, sicker veterans, and thus their comorbidity burden was markedly lower. Notably, in the group with the sickest individuals (that is those with the highest comorbidity scores, who were very disabled), receiving guideline-concordant care was associated with improved survival in adjusted models compared with those patients receiving only moderate levels of care.

Keywords: schizophrenia, veterans, quality of health care, mortality, primary care, preventive care

\footnotetext{
* Correspondence: jacktsan@gmail.com

'VISN 17 Center of Excellence for Research on Returning War Veterans, Department of Veterans Affairs, 4800 Memorial Drive (151C), Waco, TX 76711, USA

Full list of author information is available at the end of the article
}

\section{Ciomed Central}

(C) 2012 Tsan et al; licensee BioMed Central Ltd. This is an Open Access article distributed under the terms of the Creative Commons Attribution License (http://creativecommons.org/licenses/by/2.0), which permits unrestricted use, distribution, and reproduction in any medium, provided the original work is properly cited. 


\section{Background}

Premature mortality among individuals with schizophrenia is well documented in the literature [1,2]. The increased mortality rates of these individuals is linked to their increased burden of medical $[3,4]$ and psychiatric [5] comorbidities. Physical disorders in individuals with schizophrenia account for an estimated $60 \%$ of premature deaths [6], yet, these individuals are less likely to seek appropriate care and may use medical services intermittently, leading to unresolved medical problems, decreased treatment adherence, and premature mortality [7], which might be preventable with appropriate care.

To target the diverse health care needs of individuals with schizophrenia, the American Psychiatric Association (APA) outlined in 2004 practice guidelines for appropriate preventive care [8]. In addition to psychiatric medication management, the APA guidelines highlight the importance of comprehensive care for common conditions including hypertension [9,10], diabetes [11], obesity [12], lipid disorders [6], pulmonary and other infections [13], cardiovascular disease and metabolic syndrome [14], tobacco and substance use disorders [15], and liver disease [16]. Whether and which of these types of care are typically accessed and how they are related to survival remains to be studied.

Previous research on older patients with schizophrenia receiving care from the Veterans Health Administration (VA) noted associations of attendance at primary-care clinics with improved odds of survival, results that led to a directive for outreach to patients with schizophrenia who fail to access care for 12 months or more $[3,17,18]$. This study examined veterans with schizophrenia age 50 or older, receiving care in the VA, to determine whether there were particular types and patterns of routine outpatient care that were associated with improved survival, using a framework of specific care recommendations, over the 8-year period from October 2001 through September 2009.

\section{Methods}

\section{Study design}

The study was approved by the local institutional review board (IRB) before initiation. As part of a larger retrospective study of late-life healthcare for veterans with chronic disease [17], veterans diagnosed with schizophrenia and receiving VA care during the fiscal year (FY) 2002 (FY02; from October 2001 to September 2002) were followed to the end of FY09 using administrative extracts from the all-electronic medical records system of the VA.

\section{Study sample}

Veterans seeking care from the VA were included in this study if they were diagnosed with schizophrenia (International Classification of Disease (ICD)9 code 295, excluding 295.5) on at least two outpatient or one inpatient dates during FY02, and were at least 50 years old in that year [19]. Exclusions were: death during FY02 $(\mathrm{n}=2,051)$, female gender $(\mathrm{n}=1,773)$, inconsistent dates with respect to death (probable data-entry errors; $\mathrm{n}=134)$, missing data on veteran status $(\mathrm{n}=125)$, and long-term inpatient status in FY02 $(150+$ days; $\mathrm{n}=24)$ [20], leaving 49,173 patients (mean \pm SD age $59.6 \pm 9.2$ years, range 50 to 102) in the cohort.

\section{Measures}

Annual care indicators corresponded to APA categories [8]. Care type was captured from VA outpatient clinic identifiers (stop codes) and care provision codes (Current Procedural Terminology (CPT) and Healthcare Common Procedure Coding System (HCPCS); see Additional file 1). Recommendations for medical care addressed: weight management, tobacco cessation, infectious diseases (HIV, hepatitis $\mathrm{C}$, or syphilis), cardiovascular risk (diabetes, hypertension, dyslipidemia, heart failure), and blood chemistries (renal and liver function, hyperprolactinemia). Eye care was also included, as older people require regular eye examinations, especially if they have co-occurring diabetes. Recommended mental health care included: individual psychotherapy, group counseling, family interventions, psychiatry medication consults, and drug/alcohol care or screening. The APA guidelines discuss the value of individual, group, and family counseling for patients with schizophrenia, emphasizing that treatment delivery must be tailored to the patient, leading to our selection of these five types of mental health care. Receipt of any care during a year constituted the meeting guidelines for that care type. The indicators were then summed to assess total annual care (range 0 to 11 for each year).

Given that antipsychotic treatment is strongly recommended for patients with schizophrenia, and medication adherence correlates with better outcomes [21], we also generated mutually exclusive covariates assessing baseline antipsychotic use: 1) good adherence was indicated by having the equivalent of 30-day prescriptions of antipsychotics (AP) for at least 10 months annually (10/12 or $83 \%$ adherence); 2) poor adherence was indicated by prescriptions for 1 to 9 months; and 3) irregular antipsychotic adherence. Irregular adherence was indicated by 0 months of AP use during the baseline year. Good adherence served as the reference group for the two indicators of suboptimal antipsychotic use. Gaps of 3 or more months in antipsychotic use ( $\leq 75 \%$ adherence) are associated with psychiatric readmission [21].

The primary outcome of interest (death) was based on the date of death as given in the VA Mini-Vital Status file, which is compiled from four sources using a validated algorithm for discordant cases. Sohn and colleagues [22] 
reported $\sim 98 \%$ sensitivity for the VA file relative to the National Death Index. Survival was assessed in days.

We also assessed age, race, ethnicity, marital status, and VA priority score at baseline (FY02). VA priority, based on disability from a condition attributed to military service, describes why a veteran qualifies for VA care, and whether they have co-payments. It is a proxy for both socioeconomic status and disease severity [23]. Priority 1 veterans have a high level of disability, are often impoverished, and incur no co-payments for care or prescription medications in the VA. We contrasted priority 1 against all other priorities for VA care.

The case mix was assessed using the Charlson [24,25] and Selim [26] comorbidity indices. The Charlson sums weights for 19 conditions that are correlated with postdischarge mortality (including myocardial infarct, dementia, diabetes, and diabetes with complications) as implemented in administrative data $[27,28]$. The Selim sums 30 chronic physical and 6 psychiatric disorders as the Selim Comorbidity Score; this measure has been validated against care utilization, costs of care, and mortality [17]. The Charlson and Selim indices do not have high correlation with each other, and capture different aspects of the patient comorbidity burden.

\section{Analysis}

Frequencies and means (standard deviations) were used to describe the baseline characteristics and types of care received each year. A cluster analysis uncovered longitudinal patterns in the level and trajectory of the 11 treatment types. For the cluster analysis, patterns among patients with complete data over the study period (survivors) were assessed, resulting in three clusters with eigenvalues of greater than 1 to denote significantly distinct patterns of care [29]. Patients with censored data were then examined in seven additional cluster analyses (one for each year after baseline) using data from all years before the year of death. For each year, the censored patterns were compared with the survivor patterns. Decedents were assigned to the survivor care cluster most similar to their pattern before death occurred; all decedent clusters had eigenvalues $>1$ (graphs of the decedent trajectories are available upon request).

We examined Kaplan-Meier estimates of the time to death or to the end of the study (data right-censored) by care cluster. Both log-rank and Wilcoxon statistics showed significant differences between the three clusters $(P<0.01)$. A Cox proportional-hazards regression model estimated risk-adjusted survival as a function of care cluster; covariates included age, Charlson and Selim comorbidity indices, medication adherence indicators, and VA priority 1 status.

\section{Results}

\section{Sample and care descriptive}

The Charlson and Selim comorbidity scores were 1.00 $(\mathrm{SD} \pm \pm 1.5$ and 3.82 ( $\mathrm{SD} \pm 2.2$, respectively, with an estimated correlation of $r=0.59$ (35\% shared variance; $\mathrm{p}<0.05)$. Slightly more than $>$ half $(53 \%)$ had VA priority 1 status (see Table 1 ).

In terms of guideline-concordant care for FY02 to FY09, most of the veterans were receiving cardiovascular care (67 to $76 \%$ ), hepatic and renal function assays (79 to $84 \%$ ), individual counseling (72 to $85 \%$ ), and psychiatry consults (66 to $82 \%$ ). Family counseling, individual counseling, group counseling, and psychiatry consults appeared to decrease over time (from $82 \%$ with psychiatric consults in FY02 to 66\% in FY09), whereas cardiovascular and drug/alcohol care appeared to increase (see Figure 1). Only a small proportion of VA patients in this study had documented care for nicotine dependence (3 to $6 \%$ ), weight management (11 to $13 \%)$, drug/alcohol use (13 to $17 \%$ ), infectious disease (22 to $28 \%$ ), or eye care (26 to $35 \%$ ), or received group (12 to $21 \%$ ) or family counseling (3 to $8 \%)$,.

\section{Cluster profiles}

Individuals with high-consistent care averaged 5.4 types of care across all years (Figure 2). This was the most common pattern, evident in the youngest group with the most comorbidity and the largest proportion of priority 1 veterans $(n=20,854)$. This group experienced $26 \%$ mortality over the follow-up period (unadjusted rate). Those with moderate-consistent care averaged 3.8 types of care, and these were a group of veterans with an unadjusted mortality rate of $36 \%(n=18,218)$. People in the poor-decreasing trajectory averaged 1.9 types of care across all years; this group had the oldest patients (but with a wider variation in age, with $\mathrm{SD}=10$ years), but had the least comorbidity and smallest proportion of priority 1 veterans, and experienced an unadjusted mortality rate of $31 \%(n=10,101)$. Cancer was relatively more common in the moderate-consistent care group whereas cardiovascular diagnoses were highest in the high-consistent care group (Table 2).

Patients in the high-consistent group consistently received more types of care over time, including weight management (18\% to $21 \%$ per year), infectious disease ( $32 \%$ to $41 \%$ ), renal/liver function ( $95 \%$ to $99 \%$ ), and individual counseling (93 94\%), compared with those in the moderate-consistent ( $6 \%$ to $8 \%, 18 \%$ to $22 \%, 80 \%$ to $88 \%$, and $74 \%$ to $82 \%$, respectively) or poor-decreasing groups ( $2 \%$ to $8 \%, 6 \%$ to $16 \%, 28 \%$ to $65 \%$, and $13 \%$ to $72 \%$, respectively). Overall, there was an apparent increasing trend from baseline to FY09 in the proportion of patients receiving cardiovascular care (from $83 \%$ to $96 \%$ ), eye care 
Table 1 Baseline characteristics of veterans with schizophrenia aged 50 years or older $(n=49,173)$.

\begin{tabular}{ll}
\hline Parameter & \\
\hline Age, years $^{1}$ & $59.6 \pm 9.2(56.0)(50$ to 102) \\
$\mathrm{SCl}^{1}$ & $3.82 \pm 2.2(3.0)(1$ to 16) \\
$\mathrm{CCS}^{1}$ & $1.00 \pm 1.5(1.0)(0$ to 16)
\end{tabular}

Ethnicity, n (\%)

White

$32,245(68.6)$

Black

$14,117(30.0)$

Hispanic

$4,591(9.3)$

Other

$658(1.4)$

Missing data

2,153

Marital status, n (\%)

Single (never married) $\quad 19,250(39.8)$

Married

$13,333(27.5)$

Divorced

Widowed

Missing data

Priority 1 status

Antipsychotic adherence, $\mathrm{n}$ (\%)

Good

Poor

Irregular

Medical/physical disorders, n (\%)

Anemia

Angina

Cancer

Cataracts

Congestive heart failure

COPD

Diabetes mellitus (per SCI)

Hepatitis

Hypertension

Dyslipidemia

Cardiac arrhythmias

Lower back pain

Myocardial infarction

Nicotine dependence

Obesity

Peripheral vascular disease

Chronic renal disease

Stroke

Urinary tract infection

Mental health disorders

Alcohol abuse or dependence

Anxiety

Bipolar disorder

Depression

PTSD

Abbreviations: CCS, Charlson Comorbidity Score; COPD, chronic obstructive pulmonary disease; SCl, Selim Comorbidity Index; PTSD, post-traumatic stress disorder.

${ }^{1}$ Data are mean \pm SD (median) (range).

$13,086(26.6)$

$27,349(55.6)$

$8,738(17.8)$

2,935 (6.0)

$1,148(2.3)$

$3,169(6.4)$

$5,279(10.7)$

$1,595(3.2)$

$7,482(15.2)$

$11,423(23.2)$

$2,786(5.7)$

$21,062(42.8)$

$14,147(28.8)$

$1,940(3.9)$

$5,872(11.9)$

$1,147(2.3)$

$11,429(23.2)$

$5,920(12.0)$

$1,399(2.8)$

$1,344(2.7)$

$1,422(2.9)$

$1,484(3.0)$

$6,448(13.1)$

4,221 (8.6)

$5,998(12.2)$

$10,488(21.3)$

$6,075(12.4)$
(37\% to $55 \%)$, drug/alcohol care (20\% to $27 \%$ ) and renal/ liver function care (95\% to $99 \%)$. However, a decreasing trend was seen for psychiatric medication consults (91\% to $88 \%$ ), group counseling ( $31 \%$ to $23 \%$ ), and family counseling (11\% to $5 \%)$. Weight management, nicotine dependence, and individual counseling appeared fairly stable across the years. A smaller proportion of patients in the poor-decreasing group received recommended treatment across all types of care than in the highconsistent and moderate-consistent groups. In the poordecreasing group, a moderate percentage of patients initially received several types of recommended care, but this proportion dropped sharply over time from baseline to FY09: cardiovascular care dropped from $48 \%$ to $24 \%$, renal/liver function care from $65 \%$ to $29 \%$, psychiatric medication management from $70 \%$ to $20 \%$, and individual counseling from $72 \%$ to $24 \%$. In the poor-decreasing group, the numbers receiving weightmanagement care (8\% to $2 \%)$ and eye care (16\% to $7 \%)$ were particularly low.

\section{Mortality models}

The relationship between receipt of guideline-concordant care and mortality rate was examined with and without adjustment for baseline characteristics, using Cox proportional-hazards models. In the unadjusted model, the estimated probability of surviving at least 5 years was 0.85 for those in the high-consistent group, 0.77 in the moderate-consistent groups, and 0.82 in the poor-decreasing group. Survival from Oct 2001 until death averaged 7.1 years for the high-consistent, 6.6 years for the moderate-consistent, and 6.9 years for the poor-decreasing group. The 25th percentile of survival time was estimated at 7.6 years for the high-consistent group, 5.5 years for the moderate-consistent group, and 6.3 years for the poor-decreasing group.

In the multivariable adjusted model, the hazard ratio (HR) for the moderate-consistent group indicated a $41 \%$ increase in the mortality rate $(\mathrm{HR}=1.41,95 \% \mathrm{CI} 1.36$ to 1.47) relative to the high-consistent group (see Table 3 ). However, patients in the poor-decreasing group experienced a $6 \%$ decrease in mortality rate $(\mathrm{HR}=0.94 ; 95 \% \mathrm{CI}$ 0.90 to 0.99 ) relative to the reference (high-consistent) group.. These results varied from those of the unadjusted survival analysis. Whereas the unadjusted survival findings showed that patients receiving consistently frequent care had the greatest survival rate (Figure 3 ), the adjusted model showed that patients in the poor-decreasing care group had a lower mortality rate. Mortality also correlated with age (increasing by $78 \%$ per decade) and comorbid disease burden. For each unit increase in the Charlson and Selim scores, mortality increased by $21 \%$ and $1 \%$, respectively. 


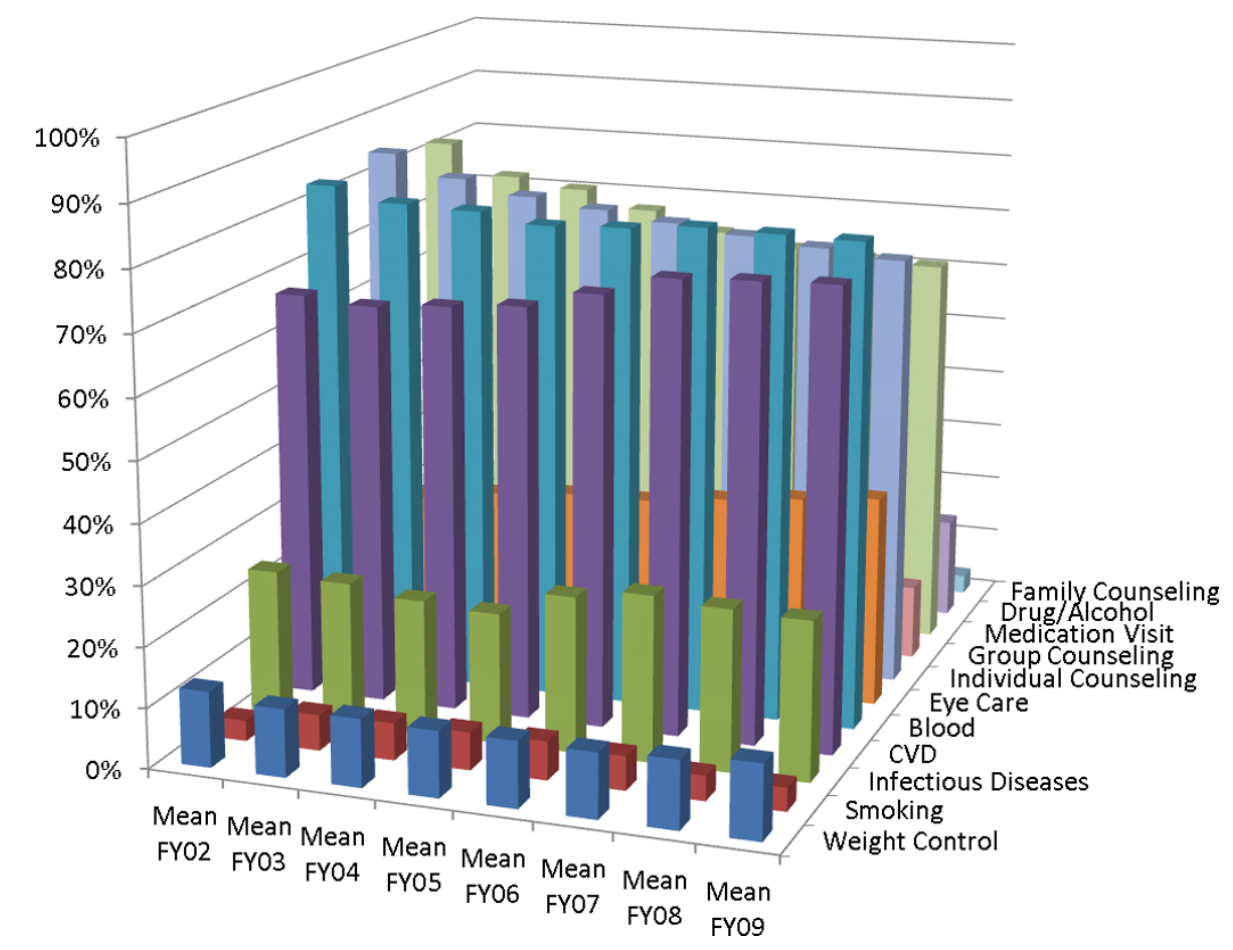

Figure 1 Proportion of patients with schizophrenia receiving each type of care, stratified by fiscal year $(n=49,173)$.

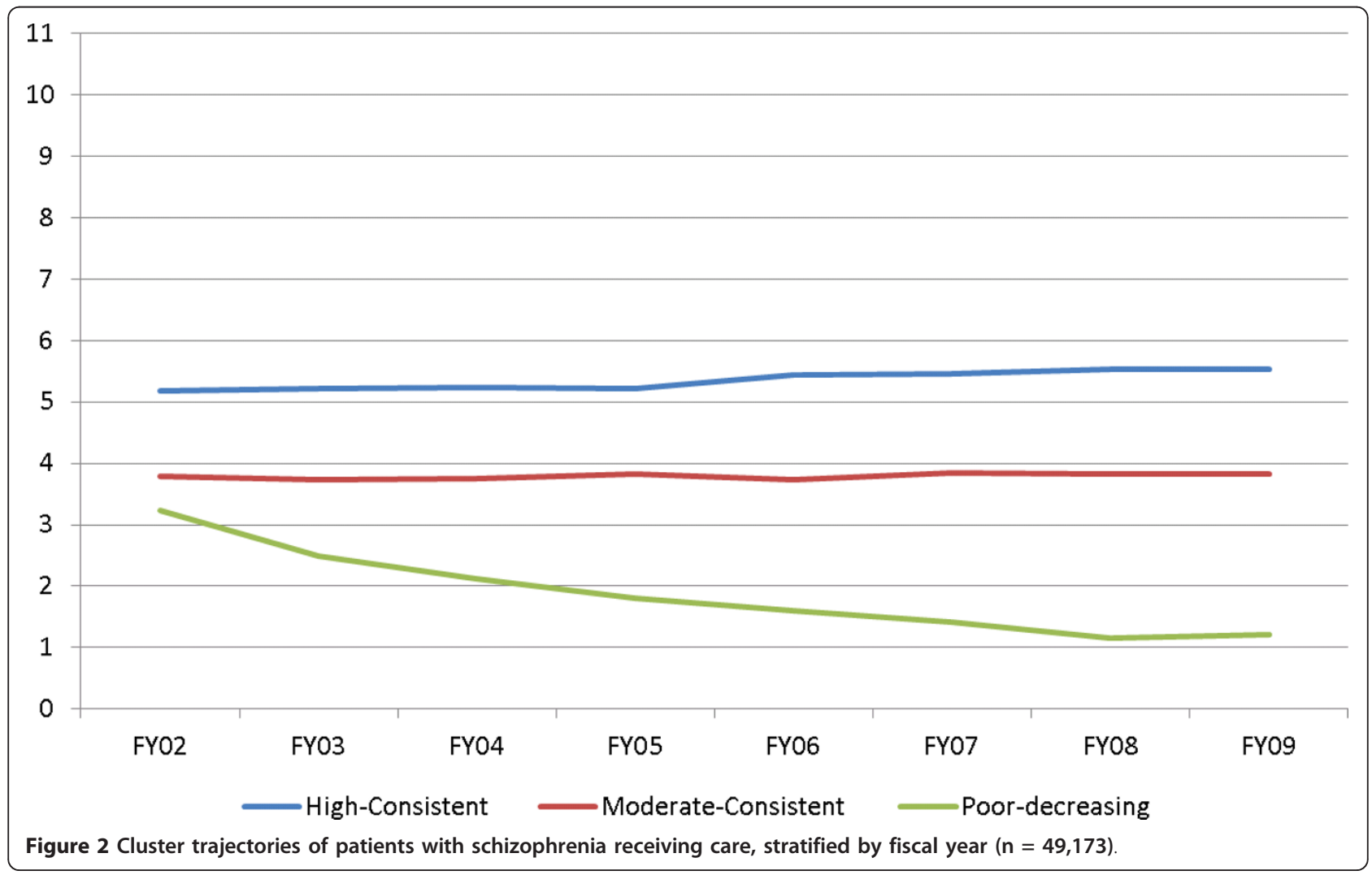


Table 2 Baseline patient characteristics by clusters $(n=49,173)$.

\begin{tabular}{|c|c|c|c|}
\hline & high-consistent care, $\mathrm{n}=20,854$ & Moderate-consistent care, $\mathrm{n}=18,218$ & poor-decreasing care, $n=10,101$ \\
\hline$\overline{\text { Age, }_{\text {years }}{ }^{1}}$ & $57.8 \pm 8.0$ & $60.3 \pm 9.5$ & $62.2 \pm 10.1$ \\
\hline $\mathrm{SCl}{ }^{1}$ & $4.37 \pm 2.3$ & $3.48 \pm 2.1$ & $3.31 \pm 2.1$ \\
\hline $\mathrm{CCS}^{1}$ & $1.16 \pm 1.6$ & $0.91 \pm 1.5$ & $0.82 \pm 1.3$ \\
\hline \multicolumn{4}{|l|}{ Ethnicity, n (\%) } \\
\hline White & $13,602(65.2)$ & $12,069(66.3)$ & $6,574(65.1)$ \\
\hline Black & $6,529(31.3)$ & $5,000(27.5)$ & $2,588(25.6)$ \\
\hline Other & $289(1.4)$ & $242(1.3)$ & $127(1.3)$ \\
\hline Hispanic & $2,518(12.1)$ & $1,513(8.3)$ & $560(5.5)$ \\
\hline Unknown & $434(2.1)$ & $907(5.0)$ & $812(8.0)$ \\
\hline \multicolumn{4}{|l|}{ Marital status } \\
\hline Single (never married) & $7,548(36.2)$ & $7,243(39.8)$ & $4,459(44.1)$ \\
\hline Married & $6,317(30.3)$ & $4,937(27.1)$ & $2,079(20.6)$ \\
\hline Divorced & $6,049(29.0)$ & $4,945(27.1)$ & $2,869(28.4)$ \\
\hline Widowed & $703(3.4)$ & $765(4.2)$ & $492(4.9)$ \\
\hline Priority 1 status & $12,232(58.7)$ & $9,616(52.8)$ & 4,339 (43.0) \\
\hline Mortality & $5,491(26.3)$ & $6,585(36.2)$ & $3,126(31.0)$ \\
\hline \multicolumn{4}{|l|}{ Antipsychotic adherence } \\
\hline Good & $6,640(31.8)$ & $4,737(26.0)$ & $1,709(16.9)$ \\
\hline Poor & $11,700(56.1)$ & $10,203(56.0)$ & $5,446(53.9)$ \\
\hline Irregular & $2,514(12.1)$ & $3,278(18.0)$ & $2,946(29.2)$ \\
\hline \multicolumn{4}{|l|}{ Medical/physical diagnoses } \\
\hline Anemia & $1,348(6.5)$ & $1,071(5.9)$ & $516(5.1)$ \\
\hline Angina & $648(3.1)$ & $348(1.9)$ & $152(1.5)$ \\
\hline Cancer & $1,481(7.1)$ & $1,237(6.8)$ & $451(4.5)$ \\
\hline Cataracts & $2,932(14.1)$ & $1,555(8.5)$ & $792(7.8)$ \\
\hline Congestive heart failure & $715(3.4)$ & $613(3.4)$ & $267(2.6)$ \\
\hline COPD & $3,526(16.9)$ & $2,753(15.1)$ & $1,203(11.9)$ \\
\hline Diabetes mellitus per Selim & $6,724(32.2)$ & $3,217(17.7)$ & $1,482(14.7)$ \\
\hline Hepatitis & $1,676(8.0)$ & $796(4.4)$ & $314(3.1)$ \\
\hline Hypertension & $10,876(52.2)$ & $7,128(39.1)$ & $3,058(30.3)$ \\
\hline Dyslipidemia & $7,999(38.4)$ & $4,547(25.0)$ & $1,601(15.9)$ \\
\hline Cardiac arrhythmias & $962(4.6)$ & $660(3.6)$ & $318(3.2)$ \\
\hline Lower back pain & $3,269(15.7)$ & $1,835(10.1)$ & $768(7.6)$ \\
\hline Myocardial infarction & $528(2.5)$ & $431(2.4)$ & $188(1.9)$ \\
\hline Nicotine dependence & $5,897(28.3)$ & $3,770(20.7)$ & $1,762(17.4)$ \\
\hline Obesity & $3,614(17.3)$ & $1,649(9.1)$ & $657(6.5)$ \\
\hline Peripheral vascular disease & $658(3.2)$ & $485(2.7)$ & $256(2.5)$ \\
\hline Chronic renal disease & $616(3.0)$ & $503(2.8)$ & $225(2.2)$ \\
\hline Stroke & $596(2.9)$ & $488(2.7)$ & $338(3.4)$ \\
\hline Urinary tract infection & $662(3.2)$ & $548(3.0)$ & $274(2.7)$ \\
\hline \multicolumn{4}{|l|}{ Mental health disorders } \\
\hline Alcohol & 3,507 (16.8) & $1,859(10.2)$ & $1,082(10.7)$ \\
\hline Anxiety & $2,210(10.6)$ & $1,357(7.5)$ & $654(6.5)$ \\
\hline Bipolar disorder & $3,163(15.2)$ & $1,796(9.9)$ & $1,039(10.3)$ \\
\hline Depression & $5,548(26.6)$ & 3,199 (17.6) & $1,741(17.2)$ \\
\hline PTSD & $3,541(17.0)$ & $1,744(9.6)$ & $790(7.8)$ \\
\hline
\end{tabular}

Abbreviations: CCS, Charlson Comorbidity Score; COPD, chronic obstructive pulmonary disease; SCl, Selim Comorbidity Index; PTSD, post-traumatic stress disorder. ${ }^{1}$ Data are mean \pm SD (median) (range). 
Table 3 Cox proportional-hazards model for mortality $(n=49,173)$.

\begin{tabular}{|c|c|c|c|}
\hline Predictor & Estimate, mean $\pm \mathrm{SE}$ & HR $(95 \% \mathrm{Cl})$ & $P$ value \\
\hline Age (in decades) & $0.58(0.01)$ & $1.78(1.75$ to 1.81$)$ & $<0.01$ \\
\hline CCS & $0.19(<0.01)$ & 1.21 (1.20 to 1.23$)$ & $<0.01$ \\
\hline SCI total & $0.01(<0.01)$ & 1.01 (1.00 to 1.02 ) & $<0.01$ \\
\hline Priority 1 status & $-0.09(0.02)$ & 0.91 (0.89 to 0.94 ) & $<0.01$ \\
\hline \multicolumn{4}{|l|}{ AP adherence } \\
\hline Poor & $0.08(0.02)$ & 1.08 (1.04 to 1.12$)$ & $<0.01$ \\
\hline Irregular & $0.15(0.02)$ & $1.16(1.10$ to 1.21$)$ & $<0.01$ \\
\hline \multicolumn{4}{|l|}{ Type of care } \\
\hline Moderate-consistent & $0.35(0.02)$ & 1.41 (1.36 to 1.47$)$ & $<0.01$ \\
\hline Poor-decreasing & $-0.06(0.02)$ & 0.94 (0.90 to 0.99$)$ & 0.02 \\
\hline
\end{tabular}

Abbreviations: AP, Antipsychotic; CCS, Charlson Comorbidity Score; HR, hazard ratio; SCl, Selim Comorbidity Index.

\section{Discussion}

For older male outpatients with schizophrenia, our analyses support a modest longitudinal association over an 8-year period between a greater scope of guideline-concordant care and survival. However, patients in the group who received the least comprehensive guideline-concordant care had better adjusted odds of survival than those receiving high or moderate levels of care, although the benefit relative to those with high-consistent care was small. This group was characterized by lower comorbidity, older age (but a larger age range), and fewer VA priority 1 veterans (those with a high level of disability), thus apparently less need for care. The mechanisms by which this survival benefit might accrue include access to care being

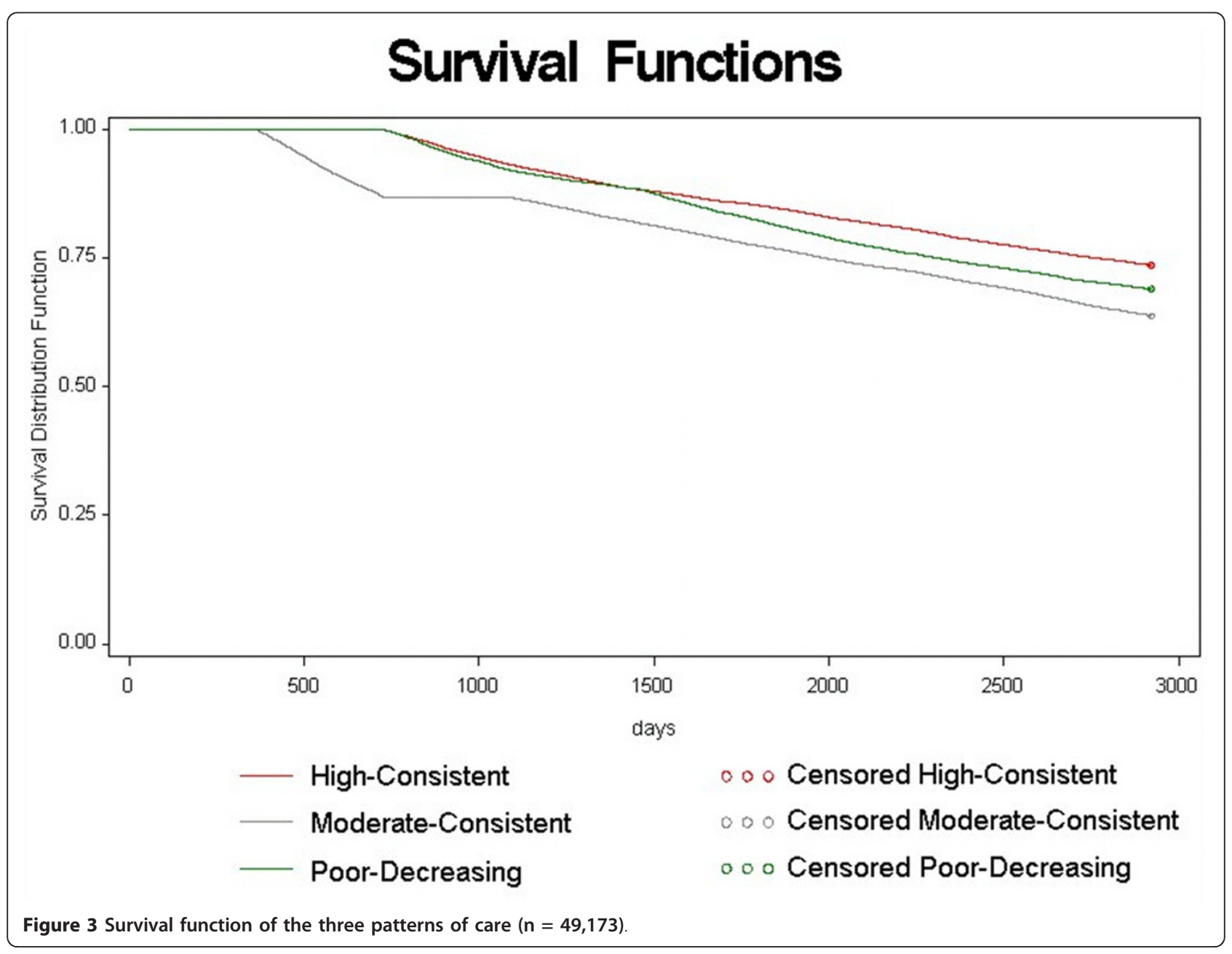


selectively used, emphasis on prevention and early disease management being common in primary care, and a focus on necessary care [30]. Possibly these patients also avoided excess medical care that might itself carry increased risk to their well-being through iatrogenic mechanisms. Moreover, patients in the poor-decreasing care group had less comorbidity, which is known to account for half the excess mortality of persons with schizophrenia [31]. Their relatively lower rates of cardiovascular diagnoses may account for some of the findings and the lower levels of care.

Interestingly, this sample of veterans with schizophrenia received on average only 4.8 out of the 11 care types examined. The patient's burden of illness translates into the healthcare system's challenge of providing appropriate preventive services; difficult care coordination for patients with the diminished self-care capacity or cognitive deficits common with schizophrenia may account for this modest level of guideline-concordant care [32]. Least common were visits for nicotine dependence, weight management, drug/alcohol dependency infectious diseases, eye care, and group psychotherapy. Over time, possibly due to aging, the percentage of patients receiving cardiovascular and eye care tended to increase, which is appropriate given the age-related nature of cardiovascular and eye disorders. The failure to address weight management is unfortunate, as this common condition potentiates much cardiovascular disease. Other research on VA patients with schizophrenia noted no disparities in receipt of obesity care practices, but did note a generally low level of weight management [33].

Of note, the proportion of patients receiving mental health treatment (psychiatric consults for medication management, and individual, family, and group therapy) gradually decreased over time. Regarding medication management, this may indicate diminishing use of antipsychotic agents in later life. A 2004 review suggested that older persons with schizophrenia do not need fewer medications, but a recent analysis suggests that they may require lower doses or antipsychotics or none at all $[34,35]$. Nevertheless, patients who used antipsychotics consistently had a longer survival than those with poor or inconsistent use, seemingly in line with the report by Maher and Theodore, who found that appropriate antipsychotic use was not associated with increased mortality [36]. The results from liver and renal function tests, often used to monitor adverse drug effects, were generally high [37]. Families involved in the care of these patients should be mindful of the need to work closely with care providers regarding medication options (for example, dose adjustment, possible medication changes to limit weight gain). Psychological counseling, particularly family counseling, can improve illness insight and thence treatment adherence, and also improve family functioning [38]. The declines in mental health counseling merit investigation into the client and family need for and benefit from these services.

Patients who fail to come in for outpatient care for 12 months or more [17] experience heightened risk of death, whether through increased risk due to heart disease [39], adverse effects of antipsychotics on cardiovascular functioning and metabolic regulation [40,41], or increased risk of liver or renal disease [16]. Over time, the proportion of patients receiving renal/liver function care and psychiatric medication management in the moderate-consistent and poor-decreasing care groups gradually declined. Receiving liver function screening, especially for patients with liver disease, is associated with appropriate treatment and longer survival [42]. Insufficient monitoring would provide fewer opportunities to make adjustments in medication regimens including hepatotoxic medications, and patients are likely to continue using psychotropic medications that exacerbate liver problems if poor liver functioning goes undetected. Accordingly, providers and family caretakers should be vigilant when patients decrease their healthcare visits over time or miss appointments.

As this is perhaps the first study to examine the association of comprehensive guideline-based care with survival for older male patients with schizophrenia, our findings suggest that greater adherence to screening and treatment recommendations is crucial to patient survival, and may reduce premature mortality. Additionally, early detection and treatment of incipient problems is likely to produce greater returns in terms of quality of life for older patients with schizophrenia, and to reduce the strain of caretaking for the older patient's social network. It is important for mental and primary healthcare practitioners to coordinate care efforts and improve overall adherence to these treatment guidelines. These patients may benefit from aggressive outreach and use of a patient-centered medical home to improve care coordination and treatment adherence.

\section{Limitations and strengths}

This study relied on administrative data, thus severity ratings of psychiatric or medical illnesses were not available and nor were quality-of-life assessments. Medicare and other out-of-system data were unavailable, thus any such services received were not captured by our study methods [23]. Future research linking VA with Medicare data to assess guideline-concordant care could determine the extent of bias introduced in our results by reliance on a single healthcare system for those persons covered by Medicare. For example, patients in the poordecreasing care cluster may have been more likely than others to seek healthcare services outside the VA after the baseline year, as many were reaching the age of 62 (early eligibility) or 65 (usual eligibility) years during the 
period of observation. On the other hand, patients with serious mental illness tend to stay within the VA [43]. Although a high proportion of patients received family counseling, it is uncertain whether this translated into evidenced-based family services for schizophrenia, considering that previous studies have identified low rates of such care [44]. Of note, the present study focused on male veterans age 50 years or older, therefore results cannot be generalized to women or younger patients. VA patients tend to have lower incomes and more health problems than the general population in the USA, which may affect their need for care and the breadth of healthcare options available to them [45].

The strengths of the study include its large scale and longitudinal design, which enabled us to follow patients over an 8-year period, and an innovative approach mapping the treatment recommendations from APA guidelines onto the indicators of care received. The study addressed a crucial and serious healthcare problem, namely, premature mortality among patients with schizophrenia. We were careful to include preventive care, such as screenings for common medical and psychiatric comorbidities, as well as treatment for these conditions. A further strength was the use of cluster analysis, which enabled a practical and interpretable comparison of patient groups characterized by different patterns of care over time.

Future research on the effects of preventive care for individuals with schizophrenia is needed to replicate and extend the results of this study. Studies are needed in other healthcare systems, and including people who lack access to consistent sources of care. Also of value would be an examination of the relative importance of the types of recommended care in reducing premature mortality. For example, through family counseling, family members of patients with schizophrenia may be more likely to assist with medical adherence and coordination. This may be especially important when patients with schizophrenia require complex coordinated care, which may be difficult to manage.

\section{Conclusions}

Older veterans with schizophrenia received only a modest number of the recommended annual health care services in the VA. Patients receiving consistent and more comprehensive guideline-concordant care had better survival than those receiving moderate-consistent levels of care, even though they had more illnesses. These findings highlight the importance of the quality and comprehensiveness of guideline-concordant preventive healthcare for older patients with schizophrenia, augmenting the literature, which has previously shown that receipt of primary care correlates with survival $[17,46,47]$. Healthcare systems should consider ways to better engage patients and their families in coordinated healthcare services. Barriers to access, treatment needs, and treatment preferences must be addressed creatively to maintain the highest quality of care for our most vulnerable patients.

\section{Additional material}

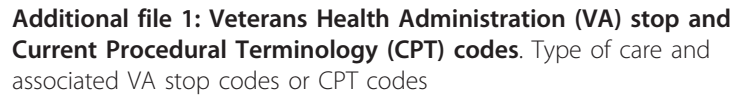

\section{Abbreviations}

APA: American Psychiatric Association; CPT: Current Procedural Terminology; FY: Fiscal year; HCPCS: Healthcare Common Procedure Coding System; HIV: Human immunodeficiency virus; IRB: Institutional review board; VA: Veterans Health Administration.

\section{Acknowledgements}

This study was funded by a grant from the Veterans Health Administration, Health Services Research and Development (number IIR-05-326; PI: Laurel A Copeland). Preparation of this report was supported by the Department of veterans Affairs Advanced Fellowship Program in Mental IIIness Research and Treatment, Central Texas veterans Health Care System (CTVHCS), and by the Center for Applied Health Research, a research center jointly sponsored by CTVHCS and Scott and White Healthcare System, Temple, TX, USA.

\section{Author details}

${ }^{1}$ VISN 17 Center of Excellence for Research on Returning War Veterans, Department of Veterans Affairs, 4800 Memorial Drive (151C), Waco, TX 76711 USA. ${ }^{2}$ VA Texas Valley Coastal Bend Health Care System, Department of Veterans Affairs, 2106 Treasure Hills Blvd., Harlingen, TX 78550, USA. ${ }^{3}$ Central Texas Veterans Health Care System, Department of Veterans Affairs, 1901 Veterans Memorial Drive, Temple, TX 76504, USA. ${ }^{4}$ Center for Applied Health Research, Scott \& White Healthcare System, 2102 Birdcreek Drive Temple, TX 76502, USA. ${ }^{5}$ Autism Comprehensive Educational Services, El Paso, TX 79912, USA. ${ }^{6}$ Department of Internal Medicine, Scott \& White Healthcare, 2401 S. 31st St., Temple, TX 76508, USA.

\section{Authors' contributions}

The idea was conceived by JT, JG, JZ, and LC. The literature search was conducted by JT, JG, JZ, and DG. ES and LC provided conceptual feedback, analysis, and design. JT, ES, LC, JG, DG, and ER reviewed and approved all CPT, HCPCS, and Stop codes. All authors read and approved the final version of the manuscript.

\section{Authors' information}

JT was affiliated with the VISN 17 Center of Excellence for Research on Returning War veterans, Department of veterans Affairs in Waco, TX at the time of the study. JT is now at VA Texas Valley Coastal Bend Health Care System at the Harlingen Outpatient Primary Care Clinic.

\section{Competing interests}

The authors on this study have no financial conflict of interests for the past 3 years. The views expressed in this article are those of the authors, and do not necessarily reflect the position or policy of the Department of veterans Affairs or the US government.

Received: 24 July 2012 Accepted: 26 November 2012 Published: 26 November 2012

\section{References}

1. Casey DA, Rodriguez M, Northcott C, Vickar G, Shihabuddin L: Schizophrenia: medical illness, mortality, and aging. Int J Psychiatry Med 2011, 41:245-51.

2. Wildgust $H J$, Hodgson $R$, Beary M: The paradox of premature mortality in schizophrenia: new research questions. J Psychopharmacol 2010, 24:9-15. 
3. Copeland LA, Zeber JE, Rosenheck RA, Miller AL: Unforeseen inpatient mortality among veterans with schizophrenia. Med Care 2006, 44:110-6.

4. Bouza C, Lopez-Cuadrado T, Amate JM: Hospital admissions due to physical disease in people with schizophrenia: a national populationbased study. Gen Hosp Psychiatry 2010, 32:156-63.

5. Weber NS, Cowan DN, Millikan AM, Niebuhr DW: Psychiatric and general medical conditions comorbid with schizophrenia in the National Hospital Discharge Survey. Psychiatr Serv 2009, 60:1059-67.

6. Lambert TJ, Velakoulis D, Pantelis C: Medical comorbidity in schizophrenia. Med J Aust 2003, 178(Suppl):S67-70.

7. Brown S, Inskip H, Barraclough B: Causes of the excess mortality of schizophrenia. Br J Psychiatry 2000, 177:212-7.

8. APA: Practice guideline for the treatment of patients with schizophrenia, 2nd Edition Arlington, VA; 2004.

9. Falissard B, Mauri M, Shaw K, Wetterling T, Doble A, Giudicelli A, De Hert M: The METEOR study: frequency of metabolic disorders in patients with schizophrenia. Focus on first and second generation and level of risk of antipsychotic drugs. Int Clin Psychopharmacol 2011, 26:291-302.

10. Meszaros ZS, Dimmock JA, Ploutz-Snyder R, Chauhan SV, Abdul-Malak Y, Middleton FA, Batki SL: Accuracy of self-reported medical problems in patients with alcohol dependence and co-occurring schizophrenia or schizoaffective disorder. Schizophr Res 2011, 132:190-3.

11. Jin $\mathrm{H}$, Meyer JM, Jeste DV: Atypical antipsychotics and glucose dysregulation: a systematic review. Schizophr Res 2004, 71:195-212

12. Allison DB, Newcomer JW, Dunn AL, Blumenthal JA, Fabricatore AN, Daumit GL, Cope MB, Riley WT, Vreeland B, Hibbeln JR, Alpert JE: Obesity among those with mental disorders: a National Institute of Mental Health meeting report. Am J Prev Med 2009, 36:341-50.

13. Copeland LA, Mortensen EM, Zeber JE, Pugh MJ, Restrepo MI, Dalack GW: Pulmonary disease among inpatient decedents: Impact of schizophrenia. Prog Neuropsychopharmacol Biol Psychiatry 2007, 31:720-6.

14. Khatana SA, Kane J, Taveira TH, Bauer MS, Wu WC: Monitoring and prevalence rates of metabolic syndrome in military veterans with serious mental illness. PLoS One 2011, 6:e19298.

15. Baker AL, Lubman DI, Hides H: Smoking and schizophrenia: treatment approaches within primary care. Primary Psychiatry 2010, 17:49-54.

16. Fuller BE, Rodriguez VL, Linke A, Sikirica M, Dirani R, Hauser P: Prevalence of liver disease in veterans with bipolar disorder or schizophrenia. Gen Hosp Psychiatry 2011, 33:232-7.

17. Copeland LA, Zeber JE, Wang CP, Parchman ML, Lawrence VA, Valenstein M, Miller AL: Patterns of primary care and mortality among patients with schizophrenia or diabetes: a cluster analysis approach to the retrospective study of healthcare utilization. BMC Health Sen Res 2009, 9:127.

18. Veterans Health Administration: Re-engaging veterans with serious mental illness in treatment. 2012, VHA Directive 2012-002, Januarry 10, 2012. Washington DC. [http://www1.va.gov/vhapublications/ViewPublication.asp? pub ID = 2476]. Accessed July 12.

19. Blow FC, McCarthy JF, Valenstein M, Austin K, Gillon L: Care for Veterans with Psychosis in the VHA, FY04: 6th Annual National Psychosis Registry Report VA National Serious Mental IIIness Treatment Research and Evaluation Center [SMITREC]: Ann Arbor, Ml; 2005.

20. Blow FC, Ullman E, Barry KL, Bingham CR, Copeland LA, McCormick R, Van Stone W: Effectiveness of specialized treatment programs for veterans with serious and persistent mental illness: a three-year follow-up. Am J Orthopsychiatry 2000, 70:389-400.

21. Valenstein M, Copeland LA, Blow FC, McCarthy JF, Zeber JE, Gillon, Bingham CR, Stavenger T: Pharmacy data identify poorly adherent patients with schizophrenia at increased risk for admission. Med Care 2002, 40:630-9.

22. Sohn MW, Arnold N, Maynard C, Hynes DM: Accuracy and completeness of mortality data in the Department of Veterans Affairs. Popul Health Metr 2006, 4:2

23. Shen Y, Hendricks A, Zhang S, Kazis LE: VHA enrollees' health care coverage and use of care. Med Care Res Rev 2003, 60:253-67.

24. Charlson M, Peterson JC: Medical comorbidity and late life depression: what is known and what are the unmet needs? Biol Psychiatry 2002, 52:226-35.

25. Charlson ME, Pompei P, Ales KL, MacKenzie CR: A new method of classifying prognostic comorbidity in longitudinal studies: development and validation. J Chronic Dis 1987, 40:373-83.

26. Selim AJ, Fincke G, Ren XS, Lee A, Rogers WH, Miller DR, Skinner KM, Linzer M, Kazis LE: Comorbidity assessments based on patient report: results from the Veterans Health Study. J Ambul Care Manage 2004, 27:281-95.

27. Deyo RA, Cherkin DC, Ciol MA: Adapting a clinical comorbidity index for use with ICD-9-CM administrative databases. J Clin Epidemiol 1992, 45:613-9.

28. Pugh MJ, Copeland LA, Zeber JE, Cramer JA, Amuan ME, Cavazos JE, Kazis LE: The impact of epilepsy on health status among younger and older adults. Epilepsia 2005, 46:1820-7.

29. Aldenderfer M, Blashfield R: Cluster Analysis Sage: Newbury Park, CA; 1984

30. Starfield B, Shi L, Macinko J: Contribution of primary care to health systems and health. Milbank Q 2005, 83:457-502.

31. Laursen TM, Munk-Olsen T, Gasse C: Chronic somatic comorbidity and excess mortality due to natural causes in persons with schizophrenia or bipolar affective disorder. PLOS One 2011, 6:e24597.

32. Goldman LS: Medical illness in patients with schizophrenia. J Clin Psychiatry 1999, 60(Suppl 21):10-5

33. Copeland LA, Pugh MJ, Hicks PB, Noel PH: Use of obesity-related care by psychiatric patients. Psychiatric Services 2012, 63:230-236

34. Schimming C, Harvey PD: Disability reduction in elderly patients with schizophrenia. J Psychiatr Pract 2004, 10:283-95.

35. Harrow M, Jobe TH, Faull RN: Do all schizophrenia patients need antipsychotic treatment continuously throughout their lifetime? A 20 year longitudinal study. Psychol Med 2012, 1-11.

36. Maher AR, Theodore G: Summary of the comparative effectiveness review on off-label use of atypical antipsychotics. J Manag Care Pharm 2012, 18: S1-20

37. Alexopoulos GS, Streim J, Carpenter D, Docherty JP: Using antipsychotic agents in older patients. J Clin Psychiatry 2004, 65(Suppl 2):5-99, discussion 100-102; quiz 103-4.

38. Giron M, Fernandez-Yanez A, Mana-Alvarenga S, Molina-Habas A, Nolasco A, Gomez-Beneyto M: Efficacy and effectiveness of individual family intervention on social and clinical functioning and family burden in severe schizophrenia: a 2-year randomized controlled study. Psychol Med 2010, 40:73-84

39. Kilbourne AM, Morden NE, Austin K, Ilgen M, McCarthy JF, Dalack G, Blow FC: Excess heart-disease-related mortality in a national study of patients with mental disorders: identifying modifiable risk factors. Gen Hosp Psychiatry 2009, 31:555-63.

40. Lieberman JA, Stroup TS, McEvoy JP, Swartz MS, Rosenheck RA, Perkins DO, Keefe RS, Davis SM, Davis CE, Lebowitz BD, Severe J, Hsiao JK: Clinical Antipsychotic Trials of Intervention Effectiveness (CATIE) Investigators. Effectiveness of antipsychotic drugs in patients with chronic schizophrenia. N Engl J Med 2005, 353:1209-23.

41. Mackin P: Cardiac side effects of psychiatric drugs. Hum Psychopharmacol 2008, 23(Suppl 1):3-14

42. Leykum LK, El-Serag HB, Cornell J, Papadopoulos KP: Screening for hepatocellular carcinoma among veterans with hepatitis $C$ on disease stage, treatment received, and survival. Clin Gastroenterol Hepatol 2007, 5:508-12.

43. Rosenheck R, Fontana A: Utilization of mental health services by minority veterans of the Vietnam era. J Nerv Ment Dis 1994, 182:685-91.

44. Lucksted A, McFarlane W, Downing D, Dixon L: Recent developments in family psychoeducation as an evidence-based practice. J Marital Fam Ther 2012, 38:101-21

45. Morgan RO, Teal CR, Reddy SG, Ford ME, Ashton CM: Measurement in Veterans Affairs Health Services Research: veterans as a special population. Health Serv Res 2005, 40:1573-83.

46. Jerant A, Fenton JJ, Franks P: Primary care attributes and mortality: a national person-level study. Ann Fam Med 2012, 10:34-41.

47. Tengs TO, Adams ME, Pliskin JS, Safran DG, Siegel JE, Weinstein MC Graham JD: Five-hundred life-saving interventions and their costeffectiveness. Risk Anal 1995, 15:369-90

\section{Pre-publication history}

The pre-publication history for this paper can be accessed here: http://www.biomedcentral.com/1741-7015/10/147/prepub

\section{doi:10.1186/1741-7015-10-147}

Cite this article as: Tsan et al:: Mortality and guideline-concordant care for older patients with schizophrenia: a retrospective longitudinal study. BMC Medicine 2012 10:147. 